\title{
The child with a non-blanching rash: how likely is meningococcal disease?
}

\author{
L C Wells, J C Smith, V C Weston, J Collier, N Rutter
}

\begin{abstract}
Aims-To examine a number of simple clinical features and investigations in children with a non-blanching rash to see which predict meningococcal infection. Methods-A total of 233 infants and children up to 15 years of age presenting with a non-blanching rash were studied over a period of 12 months. Clinical features and laboratory investigations were recorded at presentation. The ability of each to predict meningococcal infection was examined.

Results-Eleven per cent had proven meningococcal infection. Children with meningococcal infection were more likely to be ill, pyrexial $\left(>38.5^{\circ} \mathrm{C}\right)$, have purpura, and a capillary refill time of more than two seconds than non-meningococcal children. Five children with meningococcal disease had an axillary temperature below $37.5^{\circ} \mathrm{C}$. No child with a rash confined to the distribution of the superior vena cava had meningococcal infection. Investigations were less helpful, although children with meningococcal infection were more likely to have an abnormal neutrophil count and a prolonged international normalised ratio. No child with a $C$ reactive protein of less than $6 \mathrm{mg} / 1 \mathrm{had}$ meningococcal infection.

Conclusions-Most children with meningococcal infection are ill, have a purpuric rash, a fever, and delayed capillary refill. They should be admitted to hospital and treated without delay. Children with a non-blanching rash confined to the distribution of the superior vena cava are very unlikely to have meningococcal infection. Measurement of $\mathrm{C}$ reactive protein may be helpful-no child with a normal value had meningococcal infection. Lack of fever at the time of assessment does not exclude meningococcal disease.

(Arch Dis Child 2001;85:218-222)
\end{abstract}

Keywords: purpura; meningococcal infection

Recent publicity surrounding meningococcal infection in children has led to an increased awareness by both doctors and parents of the symptoms and signs. The appearance of a petechial or purpuric skin rash which does not blanch when pressed with a glass is a sign which has been particularly publicised and is widely known. While this is an important and recognisable feature, many children with such a rash do not have meningococcal infection. However, many are admitted to hospital and treated with antibiotics. General paediatricians, accident and emergency departments, and general practitioners who see the children first need guidance on recognising those who are likely to have meningococcal infection.

The aim of this study was to determine whether it is possible to predict which children with a non-blanching rash do or do not have meningococcal infection, based on the characteristics of the rash, other physical signs, and simple laboratory investigations at the time of presentation.

\section{Methods}

SUBJECTS

We prospectively enrolled all infants and children aged 15 years or less with a nonblanching rash who presented to our children's accident and emergency department over a 12 month period from 1 November 1998 to 31 October 1999 (either self or general practitioner referral). The department is the only one in the city of Nottingham and serves the children from a population of about 800000 (a paediatric population of 135000$)$. All patients with a non-blanching rash were included. We defined petechiae as non-blanching spots in the skin, less than $2 \mathrm{~mm}$ in diameter, and known to be new in onset. The lesions were classed as purpura if they were more than $2 \mathrm{~mm}$ in diameter.

\section{METHOD}

The study was entirely observational — care was determined by the on-call paediatric medical team. A member of the paediatric medical team collected the data in the children's accident and emergency department, entering it on a standard proforma at the time of presentation of the child. The following data were recorded: presenting symptoms and signs, including axillary temperature, blood pressure (hypotension was defined as $2 \mathrm{SD}$ or more below the mean for age), capillary refill time (defined as normal if less than 2 seconds), ${ }^{1}$ and details of the rash (size and distribution). Children were also characterised as being either well (smiling or crying but consolable) or ill (toxic, irritable and crying inconsolably, or lethargic).

The following investigations were sent: full blood count and differential white cell count, clotting studies (international normalised ratio (INR) and activated partial thromboplastin time ratio (APTTR)), $\mathrm{C}$ reactive protein (CRP), blood culture, and polymerase chain reaction $(\mathrm{PCR})^{2}$ for meningococcal DNA. Cerebrospinal fluid (CSF) was sent for microscopy, bacterial and viral culture, PCR, glucose, and protein when a lumbar puncture was 
Table 1 Infection with $N$ meningitidis

\begin{tabular}{ll}
\hline & $\begin{array}{l}\text { Number of cases } \\
(n=24)\end{array}$ \\
\hline Serogroup of N meningitidis & 12 \\
B & 11 \\
C & 1 \\
Unknown & 24 \\
Total & \\
Method of diagnosis & \\
Positive blood culture alone & 5 \\
$\quad$ PCR not done in 3) & \\
Positive PCR alone & $\quad 1$ blood culture contaminated) \\
Positive PCR and blood culture & 9 \\
Positive PCR, blood culture, and CSF & 1 \\
\hline
\end{tabular}

clinically indicated. Proformas were completed at the time for 197 patients; 21 (9.8\%) were completed retrospectively from the case notes after patients were identified by cross checking during or at the end of the study period. Meningococcal infection was defined using the PHLS Communicable Disease Surveillance Centre enhanced surveillance for meningococcal disease definition of a positive blood, CSF, or skin culture for Neisseria meningitidis, Gram negative diplococci in CSF, or positive PCR for meningococcal DNA from blood or CSF. The study was approved by the Queens Medical Centre Ethics Committee.

ANALYSIS AND STATISTICS

We compared children who had proven meningococcal disease with those who did not, using univariate analysis. Numbers were too small for multivariate analysis. Odds ratios (OR) with 95\% confidence intervals (CI) were calculated for a range of variables; $95 \%$ confidence intervals for clinical features or investigations which were never observed in the series were calculated by Altman's formula. ${ }^{3}$ Specificity, sensitivity, and negative and positive predictive values for a range of variables were also calculated to determine those which most usefully predict meningococcal disease.

\section{Results}

Over the 12 months of the study, there were 35918 attendances to the children's accident and emergency department, of which 9239 were for a medical condition. A total of 233 $(2.5 \%)$ children who presented to the department had a petechial or purpuric rash. We excluded 15 children who had a clear alternative diagnosis (11 with Henoch-Schonlein purpura, one with idiopathic thrombocytopenic purpura, one with haemolytic uraemic syndrome, one with acute leukaemia, and one with a previously recognised clotting disorder), leaving a total of 218 study children. Twenty four of the 218 children $(11 \%)$ had proven meningococcal disease. Table 1 shows the serogroups of the meningococci and mode of diagnosis. A further four children had possible meningococcal disease with a non-blanching rash. Two had raised antibody titres to meningococcal outer membrane proteins with a greater than fourfold rise in convalescent titres, and one had a positive throat swab. A fourth child with a widespread purpuric rash required ventilation and inotropic support. She had received intramuscular benzylpenicillin before arrival and her blood culture, PCR, serology, and throat swabs were negative. Since the diagnosis was unproven, these children were all included in the non-meningococcal group for analysis. No child had laboratory confirmation of bacteraemia with any other bacteria. Eight children $(3.7 \%)$ did not have blood cultures taken - they were not were treated with antibiotics and did not develop signs of sepsis. Six children were admitted with proven invasive meningococcal infection (five with meningitis) in the same 12 month period but did not have a non-blanching rash.

Neither season nor age was useful in predicting meningococcal infection. Fifty five per cent of children with a non-blanching rash were less than 3 years old (median age 2 years) and meningococcal infection was also more common in younger children (median age 2 years). More children with a non-blanching rash were seen in the winter months (December to February) than in the other seasons $\left(\chi^{2}\right.$; $\mathrm{p}<0.001)$; although meningococcal disease was more common in the winter months, this was not statistically significant $\left(\chi^{2} ; \mathrm{p}=0.3\right)$. A total of 184 children (84\%), including all 24 who were later proven to have meningococcal infection, were admitted to hospital for a median time of 24 hours. One child who was clinically well was admitted to hospital but discharged with no treatment-blood culture grew $N$ meningitidis at 48 hours. She was well when she was recalled and repeat blood cultures prior to initiation of treatment were negative. A total of 101 children (46\%) received antibiotics (96\% intravenous, $4 \%$ oral). No child was sent home from the accident and emergency department and subsequently readmitted with meningococcal infection. One child died from meningococcal infection during the study period.

Children with meningococcal infection were more likely to be ill (OR 16.7; 95\% CI 5.8 to 47.6), to have an axillary temperature $>38.5^{\circ} \mathrm{C}$ (OR 8.0; 95\% CI 2.7 to 23.8), purpura (OR $37.2 ; 95 \%$ CI 11.7 to 118.3 ), and a capillary refill time of more than two seconds (OR 29.4; $95 \%$ CI 9.4 to 92.6 ) than non-meningococcal children, although a substantial minority of children without meningococcal disease showed these features (table 2). Hypotension was more common in those with meningococcal disease but blood pressure was only measured in a third of all children. It is likely that these were selectively more unwell. No child with a rash confined to the distribution of the superior vena cava (head, neck, and chest above the nipple line) (74/218) had meningococcal infection.

Children with meningococcal infection were more likely to have an abnormal neutrophil count (OR 2.7; 95\% CI 1.1 to 6.5 ) and a prolonged INR (OR 30.0; 95\% CI 9.9 to 91.0 ). However, a substantial minority of children without meningococcal disease also showed these features (table 3 ). No child with a CRP of less than $6 \mathrm{mg} / \mathrm{l}(90 / 183)$ had meningococcal infection. 
Table 2 Clinical features

\begin{tabular}{|c|c|c|c|}
\hline $\begin{array}{l}\text { Variable } \\
(\% \text { recorded })\end{array}$ & $\begin{array}{l}\text { Non-meningococcal } \\
(n=194)\end{array}$ & $\begin{array}{l}\text { Meningococcal } \\
(n=24)\end{array}$ & $\begin{array}{l}\text { Odds ratio } \\
(95 \% \text { CI) }\end{array}$ \\
\hline \multicolumn{4}{|l|}{ Health $(100 \%)$} \\
\hline Well & $158(97 \%)$ & $5(3 \%)$ & \\
\hline Ill & $36(65 \%)$ & $19(35 \%)$ & 16.7 (5.8 to 47.6$)$ \\
\hline \multicolumn{4}{|l|}{ Size of rash $(100 \%)$} \\
\hline Petechiae only & $171(98 \%)$ & $4(2 \%)$ & \\
\hline Purpura too & $23(53 \%)$ & $20(47 \%)$ & $37.2(11.7$ to 118.3$)$ \\
\hline \multicolumn{4}{|l|}{ Distribution } \\
\hline SVC only & $74(100 \%)$ & $0(0 \%)$ & $0(0$ to $4 \%)$ \\
\hline Rash beyond SVC & $120(83 \%)$ & $24(14 \%)$ & \\
\hline \multicolumn{4}{|l|}{ Temperature $(100 \%)$} \\
\hline Normal $\left(<37.5^{\circ} \mathrm{C}\right)$ & $106(95 \%)$ & $5(5 \%)$ & \\
\hline $37.5-38.5^{\circ} \mathrm{C}$ & $51(91 \%)$ & $5(9 \%)$ & $2.1(0.58$ to 7.5$)$ \\
\hline$>38.5^{\circ} \mathrm{C}$ & $37(73 \%)$ & $14(27 \%)$ & $8.0(2.7$ to 23.8$)$ \\
\hline \multicolumn{4}{|l|}{ Blood pressure (39\%) } \\
\hline Normal & $66(84 \%)$ & $13(16 \%)$ & \\
\hline Hypotension & $2(29 \%)$ & $5(71 \%)$ & $12.7(2.2$ to 72.5$)$ \\
\hline \multicolumn{4}{|c|}{ Capillary refill time (99.5\%) } \\
\hline Less than 2 seconds & $165(98 \%)$ & $4(2 \%)$ & \\
\hline Over 2 seconds & $28(58 \%)$ & $20(42 \%)$ & 29.4 (9.4 to 92.6$)$ \\
\hline
\end{tabular}

SVC, superior vena cava.

Table 3 Investigations

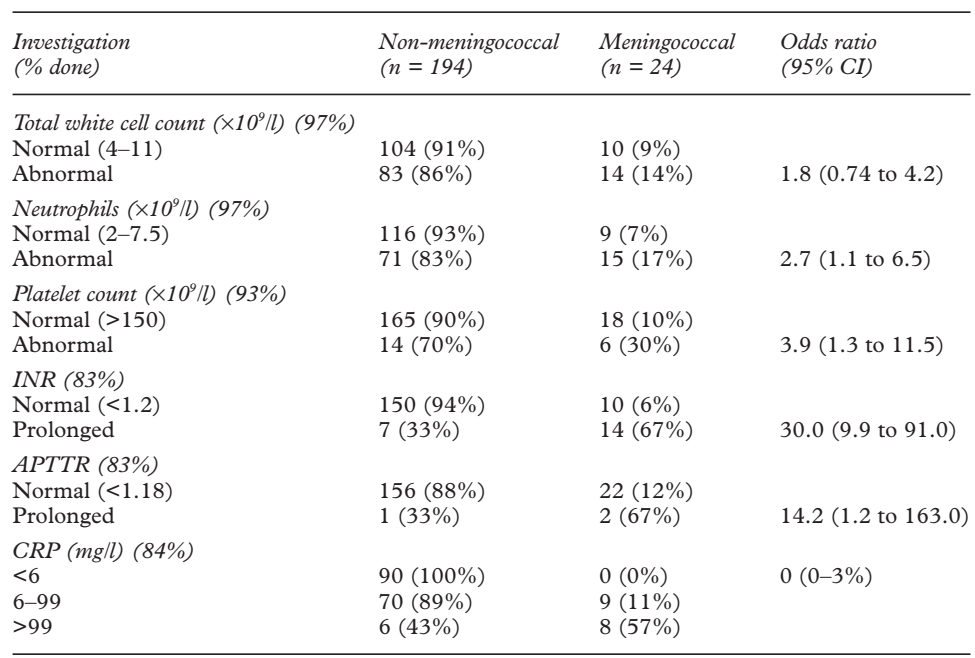

Table 4 Ability of the clinical findings to predict meningococcal infection

\begin{tabular}{|c|c|c|c|c|}
\hline Variable & $\begin{array}{l}\text { Sensitivity } \\
\%\end{array}$ & $\begin{array}{l}\text { Specificity } \\
\%\end{array}$ & $\begin{array}{l}P P V \\
\%\end{array}$ & $\begin{array}{l}N P V \\
\%\end{array}$ \\
\hline Illness & 79 (63 to 95$)$ & $81(76$ to 87$)$ & 35 (22 to 47$)$ & 97 (91 to 100$)$ \\
\hline Purpura & $83(68$ to 98$)$ & $88(84$ to 93$)$ & $47(32$ to 61$)$ & $98(92$ to 100$)$ \\
\hline Rash beyond SVC & $100(94$ to 100$)$ & $38(31$ to 45$)$ & $17(11$ to 23$)$ & $100(91$ to 100$)$ \\
\hline Fever $>38.5^{\circ} \mathrm{C}$ & $58(39$ to 78$)$ & $81(75$ to 86$)$ & $27(15$ to 40$)$ & $94(88$ to 100$)$ \\
\hline Fever $>37.5^{\circ} \mathrm{C}$ & 79 (63 to 95$)$ & 55 (48 to 62$)$ & $18(11$ to 25$)$ & 95 (88 to 100$)$ \\
\hline Hypotension & $28(7$ to 48$)$ & 97 (93 to 100$)$ & 71 (38 to 100$)$ & $84(75$ to 92$)$ \\
\hline Capillary refill $>2$ seconds & 83 (68 to 98$)$ & $85(81$ to 90$)$ & $42(28$ to 56$)$ & $98(92$ to 100$)$ \\
\hline
\end{tabular}

$95 \% \mathrm{CI}$ in parentheses.

PPV, positive predictive value; NPV, negative predictive value; SVC, superior vena cava.

Table 5 Ability of the investigations to predict meningococcal infection

\begin{tabular}{|c|c|c|c|c|}
\hline Variable & $\begin{array}{l}\text { Sensitivity } \\
\%\end{array}$ & $\begin{array}{l}\text { Specificity } \\
\%\end{array}$ & $\begin{array}{l}P P V \\
\%\end{array}$ & $\begin{array}{l}N P V \\
\%\end{array}$ \\
\hline Abnormal white count & 58 (39 to 78$)$ & $56(48$ to 63$)$ & $14(7$ to 21$)$ & 91 (84 to 99$)$ \\
\hline Abnormal neutrophil count & $68(49$ to 88$)$ & $62(55$ to 69$)$ & $17(9$ to 25$)$ & 94 (87 to 100$)$ \\
\hline $\mathrm{INR}>1.2$ & $58(39$ to 78$)$ & $96(92$ to 99$)$ & $67(47$ to 87$)$ & $94(88$ to 100$)$ \\
\hline APTTR > 1.18 & $9(0$ to 19$)$ & 99 (98 to 100$)$ & $67(13$ to 100$)$ & $88(82$ to 94$)$ \\
\hline Platelets $<150 \times 10^{9} / 1$ & $25(8$ to 42$)$ & $92(88$ to 96$)$ & $30(10$ to 50$)$ & $90(84$ to 96$)$ \\
\hline $\mathrm{CRP}>6 \mathrm{mg} / 1$ & $100(96$ to 100$)$ & $54(47$ to 62$)$ & $18(10$ to 26$)$ & $100(92$ to 100$)$ \\
\hline
\end{tabular}

$95 \% \mathrm{CI}$ in parentheses.

PPV, positive predictive value; NPV, negative predictive value.
Several of the clinical features and investigations were sensitive but poorly specific, while others were more specific but insensitive as predictors of meningococcal infection (tables 4 and 5). A rash confined to the distribution of the superior vena cava and a normal CRP each had a negative predictive value of $100 \%$ but no feature had a high positive predictive value. If the data are reanalysed, classing the four suspected cases described above as having meningococcal rather than non-meningococcal disease, the $100 \%$ negative predictive value and sensitivity of a rash in the superior vena cava distribution and a normal CRP are unchanged. Purpura, delayed capillary refill, hypotension, abnormal INR, and an abnormal neutrophil count become more specific but no less sensitive as predictors of meningococcal disease.

\section{Discussion}

We have shown that $11 \%$ of all children who presented with a petechial rash had meningococcal infection. Our incidence of meningococcal infection is high, reflecting the known recent increase in the UK. ${ }^{4}$ The study was carried out just before the national immunisation campaign against meningococcal $\mathrm{C}$ infection was launched. Assuming that the campaign is effective, our study predicts that this incidence figure will halve. This has implications for the predictive values as the negative predictive value of our observations will increase and the positive predictive value will decrease if the incidence of meningococcal disease falls. Eighty nine per cent of children did not have meningococcal infection. In $34 \%$ of children who presented with a petechial rash, the lesions were confined to the distribution of the superior vena cava. The rash is probably caused by raised venous and capillary pressure resulting from coughing, vomiting, or crying. The finding has been noted by others. ${ }^{56}$ Most of these children were well and none had meningococcal infection. In the remaining $55 \%$ of children with a petechial rash beyond the SVC distribution but without meningococcal infection, the rash was presumably caused by vasculitis or disordered coagulation in association with other non-bacteraemic bacterial or viral infections.

Other investigators have examined the incidence of meningococcal disease in children with fever and petechiae but not petechiae alone. Nguyen et al studied 129 children with fever and petechiae in New York in 1984 by retrospective case note review. ${ }^{7}$ Twenty per cent had culture proven bacterial infections, $11 \%$ with $N$ meningitidis. No single laboratory investigation was sufficiently sensitive to detect all patients with life threatening bacterial infections, but a combination of normal laboratory findings was highly specific for those without significant disease. Baker et al studied 190 children with fever and petechiae prospectively over 12 months in Cincinnati in 1989. ${ }^{8}$ Seven per cent had meningococcal disease. They concluded that patients with petechiae on their lower extremities were more likely to have invasive disease. Those with petechiae above 
the nipple line only were unlikely to have invasive disease. No variable was sensitive enough to detect all patients with serious disease. Mandl et al conducted a prospective study of 411 children presenting with fever and petechiae to a large emergency department in Boston over 18 months. ${ }^{9}$ Only five had proven sepsis, two with $N$ meningitidis, a very low incidence. They concluded that in their population, children with fever and petechiae who appeared well could be treated as outpatients because the few children with bacterial sepsis were all ill. Kuppermann et al compared clinical and haematological features of children with undiagnosed meningococcal disease to other febrile children. ${ }^{10}$ They found no clinical or haematological feature to be routinely helpful in the diagnosis of unrecognised meningococcal disease.

Brogan and Raffles recently reported the findings in 55 children presenting with fever $\left(>37.4^{\circ} \mathrm{C}\right)$ and a petechial rash, three of whom had meningococcal disease. ${ }^{11}$ They proposed the "ILL" criteria (irritability, lethargy, and low capillary refill time) in association with an abnormal total white cell count and abnormal CRP. Our study supports their suggestion that illness and delayed capillary refill time are predictors of meningococcal disease, but not their findings with regard to an abnormal total white cell count. Brogan and Raffles included only children presenting with a temperature $>37.4^{\circ} \mathrm{C}$ in association with a petechial rash, suggesting that fever is an essential component of the illness. Other studies have reported on the combination of fever and rash too. ${ }^{7-10}$ Our study is the first to be based on the presence of a non-blanching rash alone, regardless of fever. Five of our children with meningococcal disease $(21 \%)$ did not have an axillary temperature of $37.5^{\circ} \mathrm{C}$ or more. Clearly they may have had fever before or after admission, but lack of fever at the time of assessment does not exclude meningococcal disease.

There are possible limitations to our study. We believe we identified all children with petechiae by cross checking the completed proformas with all investigations sent for a petechial rash and all admissions for a petechial rash. It is possible, though, that a child who was completely well with a petechial rash may have been sent straight home without being identified. It is very unlikely that any cases of meningococcal infection were missed, as all the blood cultures from the Nottingham area are processed through the same laboratory and our cases were cross checked with these. It is possible, though unlikely, that a child who was discharged, later presented further afield. Our children's intensive care unit is the referral centre for the surrounding area and any ill child with meningococcal sepsis would have been referred there. Although a high percentage of children were investigated, data were incomplete, limiting some of the conclusions. Data collection was by different grades of doctor (junior house officer $10 \%$, senior house officer $42 \%$, and specialist registrar $48 \%$ ) - ideally this would have been carried out by a single investigator. However, it means that the study reflects actual practice rather than an ideal.

We have shown that clinical features are useful predictors of meningococcal disease. Of 218 children with a petechial rash, only one (3\%) who was well had meningococcal disease compared with $35 \%$ who were ill at presentation. Forty seven per cent of children with purpura $(>2 \mathrm{~mm}$ ) had meningococcal disease compared with $2 \%$ of children with petechiae alone. Forty two per cent of children with a prolonged capillary refill time had meningococcal disease compared with only $2 \%$ of those with a normal time. Low blood pressure was a late sign - only five children with meningococcal disease were hypotensive. As discussed above, fever was a less helpful indicator as $21 \%$ of those with meningococcal infection were afebrile $\left(<37.5^{\circ} \mathrm{C}\right)$, although meningococcal disease was more likely in those with an axillary temperature $>38.5^{\circ} \mathrm{C}$.

Our findings suggested that a child presenting with petechiae in the superior vena cava distribution is unlikely to have meningococcal disease. No child who presented with petechiae in the superior vena cava distribution alone had meningococcal disease-there is a $95 \%$ chance that only $0-5 \%$ of children with such a rash will have meningococcal disease. These children accounted for $34 \%$ of the total. Those with purpura, ill appearance, increased capillary refill time, or hypotension should be admitted and treated for meningococcal infection without delay. This accounts for a further $36 \%$. By our estimates, $70 \%$ of these will not have meningococcal disease but $96 \%$ of meningococcal infection lies within this group. The other $4 \%$ of children with meningococcal infection lay within the remaining $30 \%$ of all the children. They were well, with or without fever, with a petechial rash which extended beyond the territory of the superior vena cava. Clinical features alone provide no guidance on their identification or management.

Investigations were less useful. Total white count did not distinguish between those with and without meningococcal disease. Abnormal neutrophil count and abnormal platelet count were slightly more useful (OR 2.7; 95\% CI 1.1 to 6.5 and $3.9 ; 1.3$ to 11.5 respectively). Clotting studies were more specific but lacked sensitivity. An abnormal INR was found in $58 \%$ of patients with meningococcal disease but only $5 \%$ of those without (OR 30.0; 95\% CI 9.9 to 91.0). A normal CRP appears to be a useful predictor of those who do not have meningococcal infection. No child with a CRP $<6 \mathrm{mg} / 1$ (our laboratory definition of normal) had meningococcal disease-there is therefore a $95 \%$ chance that only $0-4 \%$ of children with a normal CRP will have meningococcal disease. We recognise the possible limitations of a normal CRP, especially in early disease. However, Marzouk et al, in a large study of 124 children with meningococcal disease, reported the diagnostic value of CRP measurement. ${ }^{12}$ Only eight of 124 patients had a CRP $<20 \mathrm{mg} / \mathrm{l}$ and of these, five patients had symptoms of less than 12 hours duration. They do not report how 
many of these had a CRP of $<6 \mathrm{mg} / \mathrm{l}$. If CRP measurement is rapidly available at all times, our study suggests that it may provide a good guide to the identification of the minority of children with meningococcal disease who are well.

Our admission rate for children presenting with a petechial or purpuric rash was $84 \%$. If we had discharged all those with a petechial rash confined to the superior vena cava distribution we would have reduced this rate to $68 \%$ (as $44 \%$ of those already discharged had a petechial rash in the superior vena cava distribution alone). If we had then discharged those remaining children who were well, with petechiae beyond the superior vena cava distribution, who did not have delayed capillary refill time or hypotension (that is, none of the features strongly associated with meningococcal disease) and who had a completely normal CRP, we would have reduced the admission rate further to $57 \%$ without sending home any child with invasive meningococcal disease.

CONCLUSION

A non-blanching rash is an important marker of meningococcal infection. If there are any of purpura, ill appearance, increased capillary refill time, or hypotension, the child should be admitted and treated for meningococcal infection without delay. If the rash is confined to the distribution of the superior vena cava, our study suggests that the child is unlikely to have meningococcal disease. In the remainder, a completely normal CRP is evidence against meningococcal infection.

We thank Dr Jenny Evennett for her help in data collection. We are also indebted to the medical and nursing staff of the general paediatric and paediatric accident and emergency departments of the Queens Medical Centre, Nottingham for their enthusiastic assistance. We would also like to thank Sarah Lewis for her help with the statistical analysis.

1 Strozik KS, Pieper CH, Roller J. Capillary refilling time in newborn babies: normal values. Arch Dis Child Fetal Neonanewborn babies: normal

2 Carrol ED, Thomson APJ, Shears P, et al. Performance characteristics of the polymerase chain reaction assay to confirm clinical meningococcal disease. Arch Dis Child 2000;83:271-3.

3 Newcombe RN, Altman DG. Proportions and their differences. In: Altman DG, Machin D, Bryant TN, Gardner MJ, eds. Statistics with confidence, 2nd edn. London: BMJ Books, 2000: 45-56.

4 Infectious diseases in England and Wales: April to June 1997. Commun Dis Rep 1998;8 (suppl 2).

5 Kravitz P. The clinical picture of 'cough purpura', a benign and non-thrombocytopenic eruption. Virginia Medical 1979;106:373-4.

6 Alcalay J, Ingber A, Sandbank M. Mask phenomenon: postemesis facial purpura. Cutis 1986;38:28.

7 Nguyen QV, Nguyen EA, Weiner LB. Incidence of invasive bacterial disease in children with fever and petechiae. Pediatrics 1984;74:77-80.

8 Baker RC, Seguin JH, Leslie N, et al. Fever and petechiae in Baker RC, Seguin JH, Leslie N, et al.
children. Pediatrics 1989;84:1051-5.

9 Mandl KD, Stack AM, Fleisher GR. Incidence of bacteraemia in infants and children with fever and petechiae. $f$ Pediatr 1997;131:398-404

10 Kuppermann N, Malley R, Inkelis SH, Fleusher GR. Clinical and hematologic features do not reliably identify children with unsuspected meningococcal disease. Pediatrics 1999; 103: 20 .

11 Brogan P, Raffles A. The management of fever and petechiae: making sense of rash decisions. Arch Dis Child 2000;83:506-7.

12 Marzouk O, Bestwick K, Thomson AP, et al. Variation in serum C-reactive protein across the clinical spectrum of meningococcal disease. Acta Paediatr 1993;82:729-33.

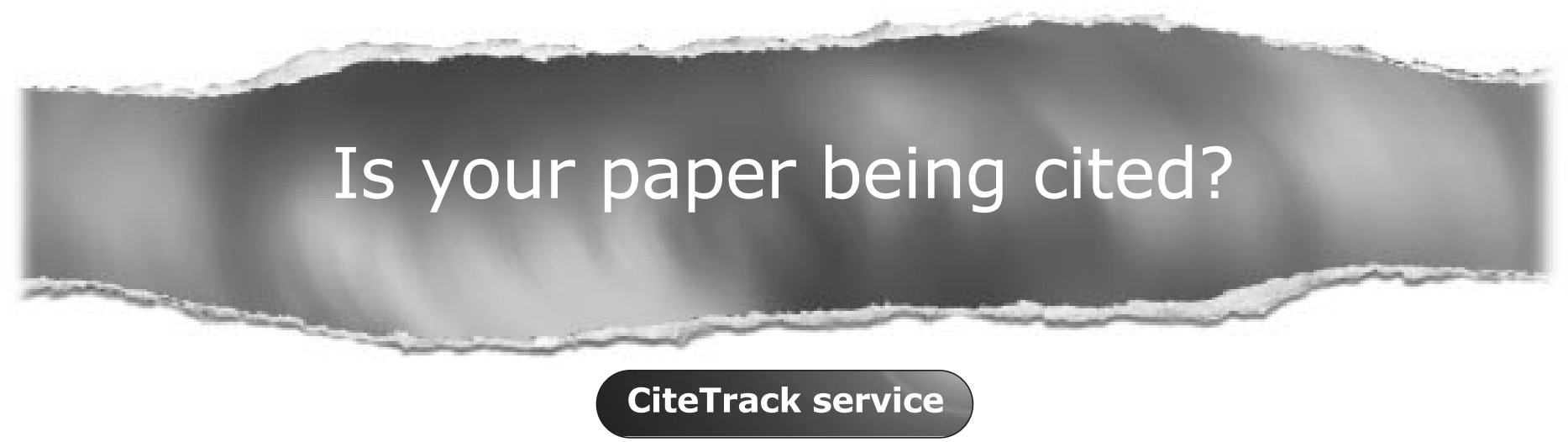

CiteTrack will alert you by email whenever new content in Archives of Disease in Childhood or a participating journal is published that matches criteria you want to track

Topics: Tell CiteTrack which words or subjects to watch for in new content Authors: Be alerted whenever key authors you are following publish a new paper Articles: Know whenever a paper of interest to you is referenced by another paper

\section{www.archdischild.com}

